\title{
What happens when counting gates can't count?
}

\section{Creating a formula to correct inaccuracies}

\author{
by Janet A. Anderson, Susan L. Durham, and Betty Dance
}

\section{$\mathrm{F}$} or years Utah State University (USU) Libraries relied on two models of entry/exit gate to provide patron-use statistics. In 1999, observations revealed that both gates undercounted the bodies passing through them by as much as $31 \%$ in a single half-hour period. This article describes the process by which a formula was derived for calculating more accurate patron counts from the inaccurate counts generated by two electronic gates.

Someone who saw a draft of this article commented, "We all know that counters as mentioned in this study are not reliable. The vendors should be held accountable for their inferior products." In fact, we do not all know that not all electronic gates can count, and one goal of this article is precisely to improve vendor accountability. The other goal is to assist libraries that already have such products in place.

Libraries collect many statistics, using them to document productivity, improvement, service, and change. Some statistics, such as reference questions asked and materials donated, are recorded and tracked by human beings. Other statistics may be recorded electronically or mechanically, including patrons passing through the library's entryway.

Libraries depend on the accuracy of statistics, not least of all to justify funding re- quests. Inaccurate statistics can cost libraries their livelihoods. Many libraries depend on mechanical and electronic gates both for security-with alarms activated when materials leave the building without the benefit of check-out-and for patron counts. But what happens when the counting gates can't count?

In 1999, USU library buildings used two models of gates, incorporating both alarm systems and patron counting. The Merrill Library building employed a single swing arm gate at the exit point (3M Security Company model 618 , installed and modified during the 1970s). This gate recorded one visitor each time a patron swung the arm aside, passed through, and allowed the arm to swing closed. Unfortunately chivalry is not dead, and courteous patrons held the arm aside for those coming behind them, as if holding open a door. During busy periods, including the hourly class change, it was common to see eight or more patrons exit at the same time, each holding the arm aside for the person behind him. The gate counted "one."

A newer alarm-and-counting combination gate is in place in the Cazier Science and Technology Library (3M Security Company model 380-4, purchased in 1995). No swing arm is involved. Patrons pass through any of four side-by-side "corridors" and are counted by an electric eye. The number of individu-

\section{About the authors}

Janet A. Anderson is campus services librarian; Susan L. Durham is statistical consu/tant, Department of Fisheries and Wildlife; Betty Dance is reference librarian at Utah State University Libraries; e-mail: janand@ngw.lib.usu.edu, betdan@ngw.lib.usu.edu; sdurham@cc.usu.edu 
Two-hour patron count:

\section{Regression of hand count on gate count}

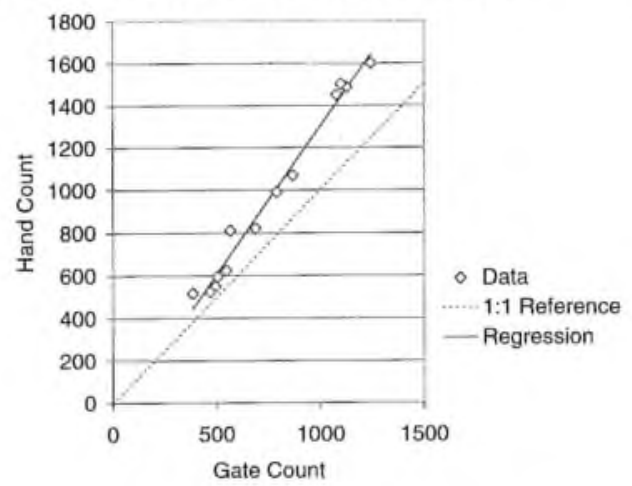

Figure 1. The reference line illustrates perfect agreement between gate and hand counts. The divergence between the reference line and the regression line illustrates the increasing error rate as patron flow increases.

als entering and exiting is recorded by a counter, installed at one end of the gate. We assumed this newer technology provided a more accurate patron count than the swing arm gate, although it seemed likely that two people passing through the same corridor together would be counted as one. Observation quickly revealed that, indeed, the electric eye could not distinguish two bodies passing through one corridor together. Further experimentation revealed that the gate count also failed when two or more people passed through different corridors at the same time.

In the spring of 1999, USU's Library Research Committee decided to collect data that would enable us to correct the inaccuracy in the statistics provided by the two gates. A brief three-hour pilot count conducted on a slow day in March revealed that while the older, swing arm gate counted between $7 \%$ and $17 \%$ low in each half-hour period, the newer gate also counted low-by between $4 \%$ and $10 \%$ over each half-hour period. (Later observations found even greater inaccuracy during busier periods.) It was also clear that the more people passing through the gate in a given half hour, the greater each gate's inaccuracy. Based on these preliminary data, we undertook a project to generate a mathematical formula that we could apply to in- crease the accuracy of our patron counts.

It is important to note that the formula we generated through the process described here is applicable only to a particular make, model, and vintage of gate, located in a particular library with its own patterns of patron flow and activity. The formula we generated cannot be assumed to apply to any other gate or library circumstance. The goal of this article is to describe the process so that other institutions can derive similar formulas appropriate to their situations.

\section{Methods}

We studied only the newer, electronic eye gate because the swing arm gate was scheduled for replacement in a matter of months. Two sampling days were selected to represent a range of heavy and light periods of traffic through the gate. Volunteer members of the University Libraries faculty and staff counted each patron passing through the gate using a hand-held tally counter.

At 15 minutes before and 15 minutes past each hour, the observer recorded the number of patrons counted by the gate and the number of patrons indicated on the tally counter. In all, 54 half-hour periods were recorded, from 7:15 a.m. to midnight on Monday, October 11, and from 7:15 a.m. to $5: 45$ p.m. (closing time is $6: 00$ p.m.) on Friday, October 15. One half-hour observation was dropped due to missing tally counter data.

Observers recorded results each half-hour as a way to increase their attention to the task and to minimize tally counter error by containing it in shorter segments. Half-hour periods also were useful because of the nature of the gate's inaccuracy (i.e., the gate is more inaccurate when there is more traffic) and because traffic through the gate is not constant, Consequently, inaccuracies of large magnitudes occur over relatively short time periods, corresponding most often to class change times.

When data are collected over longer periods, the boom and bust traffic patterns are blurred and cannot be modeled as accurately. However, the circulation department routinely collects gate count data for two-hour periods, and we needed a formula that could be applied to data collected previously. Prior to 
analysis, the half-hour data were cumulated into two-hour periods.

\section{Data analysis}

Our objective was to determine a more accurate patron count based on the easily acquired gate count. Plotting the hand (tally counter) count against the gate count (Figure 1) shows that two-hour gate counts are consistently lower than hand counts, and that the discrepancy between hand and gate counts increases as the flow of patrons through the gate increases. The plot also shows that the relationship between hand and gate counts can reasonably be assumed to be linear, and a tine describing that relationship can be obtained using linear regression. We fit a linear regression of hand counts on gate counts and obtained the following equation:

\section{hand count $=(-91.9)+(1.4 \times$ gate count $)$}

The regression is significant at $p<0.001$ $(F=628.0$ with 1 and 11 degrees of freedom; mean square error $=3251$ ) .

The regression line and the reference line (representing perfect agreement of hand and gate counts) intersect when the two-hour gate count equals 230 . Because we can safely assume that the actual count is at least as large as the gate count, we use the observed gate count as our estimate of actual count when the gate count is less than 230 .

When the gate count is 230 or greater, we estimate the actual count using the regression equation. This corrective formula can be applied to gate-generated counts regardless of time, day, week, month, semester, or year.

We emphasize that this estimation procedure is applicable only to one particular gate and patron flow, and only to gate counts recorded in two-hour periods. The formula is not applicable to any time period longer or shorter than two hours. To correct gate errors for a whole day, an estimate of actual count for each two-hour period in that day must be individually calculated and the results totaled.

It is a simple matter to write a computer routine to make the required computations based on the data already recorded by our circulation department. And basing such calculations on thousands of two-hour periods will ultimately result in greater accuracy than if the calculations were based on day-, week-, or month-long data collection.

\section{A new gate installed}

In early 2000, Merrill Library's older swing arm gate was replaced with the same make and model gate we had analyzed in the Cazier Library the previous fall. We repeated the study process with the new gate and, to our surprise, found it to be much more accurate. A phone call to $3 \mathrm{M}$ confirmed that the company had made improvements to the gate's counting system.

Our data revealed that although the new gate is more accurate than the older gate, it still fails to produce an accurate count, especially as the traffic flow increases. We calculated a second corrective formula, which is now routinely applied to the data collected by the newly installed gate.

Remember that the two gates studied are the same make and model-both inaccurate, but requiring different corrective formulas. To compare the accuracy of the two gates, imagine a two-hour period during which the gates each record 1,000 patrons passing in or out of the building. Using the formulas derived from our study data, a more accurate two-hour count for the Cazier Library would be 1,308 patrons, while the corrected count for Merrill Library would be 1.033 patrons.

\section{Conclusion}

Perhaps the ultimate counting solution would be to install three-arm turnstiles, mechanical turnstiles through which only one person can pass at one time. Counting patrons, however, is not the only function of the gates our university libraries use. Another critical function of these products is the alarm that notifies the library when materials are leaving the building without being checked out. Each library must determine the relative value of the alarm and counting functions to its own situation

We hope our experience and research alerts library and other gateway customers to some important product issues that should be considered before purchasing any patron counting system.

We also hope that our work will provide some guidelines to help other institutions evaluate the effectiveness of their electronic gateways and, if needed, to develop formulas to correct for the inaccuracies they find. 